\title{
RAPPORT
}

\section{Rapport sur l'élevage à Madagascar}

\section{CONSIDÉRATIONS GÉNÉRALES}

D'une superficie d'environ 600.000 kilomètres carrés, l'île de Madagascar est située dans l'Océan Indien entre les douzième et vingt-cinquième parallèles sud, à environ 400 kilomètres au large de la Côte Orientale d'Afrique.

Au point de vue climat (et plus spécialement en ce qui concerne l'élevage) on peut considérer cinq zones différentes à Madagascar :

I0 $^{\circ}$ la zone côtière orientale, à climat chaud et humide toute l'année;

$2^{\circ}$ La zone côtière sccidentale à climat chaud et sec, comportant une saison sèche plus marquée de mars à octobre, saison relativement fraîche;

3. La zone des hauts-plateaux s'étendant entre la côte Est et la côte Ouest, sorte d'épine dorsale et ligne de séparation des eaux entre l'Est et l'Ouest. L'altitude des hauts-plateaux dépasse souvent 2.000 mètres. La température est peu élevée et dans certaines régions, les gelées ne sont pas rares de juillet à septembre. Les pluies y sont abondantes d'octobre à mars.

$4^{\circ}$ Une zone Nord peu étendue et ntermédiaire comme climat entre la côte Est et la côte Ouest.

$5^{\circ}$ Une zone Sud à climat semi-désertique située en dehors des tropiques.

A part la zone orientale où les élevages en milieu naturel sont difficiles du fait du climat ct do la prósenco de la forêt équatoriale, toutes les régions de Madagascar sont propices à l'élevage.

A savoir :

- Les zones Ouest et Nord plus spécialement propices à l'élevage du zébu:

- Les hauts-plateaux spécialement désignés pour l'élevage du porc et des animaux améliorés.

- La zone Sud propre à l'élevage du zébu, du mouton indigene et de la chère.

Avant d'en terminer avec les considérations générales, il est bon d'indiquer que la position insulaire de Madagascar et la vigilance des Services vétérinaires ont jusqu'ici permis d'y éviter les grandes épizooties mondiales.

\section{ÉLEVAGE DU BGUF}

Le zébu malgache est certainement le Bos indicus et on peut assurer, d'après ce qui a été vu à Lucknow, qu'il ressemble en plus grand à la race « Ponward».

Il a été impossible jusqu'ici de trouver des spécimens de zébus autochtones dans les fouilles paléontologiques, aussi sommes-nous amenés à dire que le zébu malgache est d'introduction relativement récente dans l'île où il a d'ailleurs trouvé un milieu lui convenant parfaitement.

Lors de la découverte de Madagascar par les Portugais, à la fin du XVe siècle, ceux-ci ont signalé le format imposant et l'embonpoint extraordinaire des bœufs à bosse rencontrés dans l'ile.

Ont-ils vu les animaux avec les yeux de navigateurs ayant tendance à tout voir en: beau à leur arrivée à terre ou tels qu'ils étaient réellement. Il est difficile de répondre à cette question. Il semble qu'aujourd'hui le zébu de Madagascar n'a plus le format imposant du XVe siècle.

Il n'existe qu'une seule race de zébu a Madagascar. C'est un animal brachycéphale, au profil droit, avec des cornes à section circulaire nettement on lyro chez le bœuf et les vieilles vaches, los oreilles sont petites et très mobiles, l'encolure est mince, le fanon très développé; la bosse volumineuse lorsque l'animal est gras est située sur le garrct. ua taille est d'environ $1 \mathrm{~m} .35$ à $1 \mathrm{~m}$. 40 pour le taureau, et $1 \mathrm{~m} .30$ pour les vaches. Le zébu est trapu, le train postérieur est moins développé que dans les races européennes par rapport à l'avanttrain.

Le poids vif est d'environ 350 à 400 kilos chez le taureau adulte et de 250 à 300 kilos chez la vache.

Le mode d'élevage indigène qui consiste à laisser les bœufs en liberté au pâturage toute l'année, a permis de former un certain nombre de variétés qui se différencient par le format et l'importance du squelette. On trouve parfois des animaux à cornes flottantes et des animaux sans cornes qui ne constituent, en aucun cas, une race ou une variété particulière. On rencontre chez le zébu toutes les robes, unie ou pie, la prédominance de l'unə ou l'autre dans certains troupəaux n'étant due qu'à la préférence du propriśtaire pour telle ou telle couleur. 
Il a été dit plus haut que le zébu est entretenu au pâturage toute l'année. Il ne. reçoit jamais aucun supplément de nourriture en saison sèche, période pendant laquelle l'animal doit se contenter de paître l'herbe des bas-fonds ou des lisières forestières en vivant sur les réserves de graisse de sa bosse.

Pareil mode de vio no dovait nullomont aider à l'amélioration du type primitif, mais il a permis de créer un animal d'une rusticité extraordinaire, capable de tirer parti d'aliments grossiers inalibiles pour des animaux plus perfectionnés. Le zébu est un animal paisible malgré sa vie comparable à celle d'un animal sauvage et dans les troupeaux, seules les vaches suitées sont parfois dangereuses. A la rusticité du zébu on peut opposer son manque de précocité. En effet, les génisses ne sont saillies que vers 2 ans et demi et un bouf n'atteint son complet développement qu'à'l'àge de 7 ou 8 ans. $\mathbb{A}$ signaler que sur une des fermes dirigées par le Service de l'Élevage, on peut obtenir des bœufs zébus pesant de 400 à 450 kilos à 5 ans, simplement en distribuant aux animaux une faible ration de fourrage à la saison sèche, gagnant ainsi deux ans sur la vie économique d'un zébu.

Avant d'exposer les aptitudes du zébu, il faut signaler que le recensement de 1948 donne les chiffres de 6.000 .000 de têtes environ pour une population humaine d'environ 4.500.000.

Dans les régions d'élevage, la composition moyenne des troupeaux est la suivante :

- Taureaux adultes : $5 \%$.

-- Jeunes castrés et taurillons de 3 à 4 ans : $12 \%$.

- Bcufs de 5 ans et au-dessus : $10 \%$.

- Femelles de 3 ans et plus : $34 \%$.

- Génisses de 1 à 2 ans : $12 \%$.

- Taurillons de 1 an à 2 ans : $12 \%$.

- Veaux mâles et femelles : $15 \%$.

\section{Aptitudes du zébu}

10 Production du lait. - La vache zébu est très mauvaise laitière dans les conditions habituelles d'entretien, quoique susceptible de fournir une quantité de lait suffisante pour élever son veau de façon convenảble. Les vaches zébu sont rarement traites, sauf dans le Sud de l'île où les indigènes prélèvent une petite quantité de lait sur los vaches suitées du troupeau pour la fabrication de l' «abobo», ou lait caillé qui fait partie de leur alimentation.

Dans toutes les autres régions d'élevage, l'indigène préfère se procurer les protéines animales dont il a besoin sous forme de viande.

$2^{\circ}$ Production de la viande. $T$ Le bouf zébu est un excellent animal de boucherie. Malgré son manque de précocité, son format relativement réduit, il n'est pas rare d'obtenir des rendements de 48 à $50 \%$ sur des animaux maigres, rendements qui peuvent atteindre 55 et même dépasser $60 \%$ sur des animaux gras et fin gras.

3o Aptitude au travail.- Depuis très longtemps, le zébu malgache a été utilisé pour piétiner les rizières, travail pénible, ayant lieu heureusement au moment où les pâturages sont abondants, ce qui permet à l'animal de récupérer rapidement. Ce n'est qu'au début de l'arrivée des Français à Madagascar, et spécialement depuis la création d'un réseau routier, vers 1904, que le zébu malgache a été utilisé pour la charrette et plus récemment encore pour la charrue et autres instruments agricoles.

A signaler que certains bœufs sont dressés pour la selle ou le bât. Pour ce faire, les éleveurs choisissent en général des animaux sans cornes qu'ils appellent «Omby Soavaly » (Bœuf cheval).

$4^{\circ}$ Aptitude à la reproduction. - Convenablement soignée, la vache est une bonne reproductrice.

\section{Utilisation du troupeau malgache}

Ces quelques donnees sur les aplitudes du zébu permettent d'exposer ce que représente le troupeau et son utilisation. Pour le Malgache, c'est, en un mot, le coffre-fort du riche et le livret de caisse d'épargne du pauvre. Mais c'est un capital qui travaille et, sauf quelques animaux spéciaux conservés pour servir à des cérémonies rituelles ou destinés à être 'sacrifiés lors des' funérailles, le troupeau est' exploité au maximum par'l'indigène.

\section{Comment?}

Il a ćté dit plus haut que l'exploitation pour le lait n'était pas importante.

Quant au bœuf de travail, il ne passe à la charrette que pour finir à l'abattoir. Le zébu de Madagascar a donc une seule fin normale : la boucherie. Dans le tableau donnant la composition du troupeau, on peut voir que $10 \%$ du cheptel sont constitues par des animaux castrés de 5 ans et plus. Si l'on ajoute à ces bœufs le dixième des vaches adultes devenant impropres à la reproduction, soit $3,4 \%$, on arrive à un disponible annuel de $13,4 \%$. En tenant compte des pertes, on peut évaluer le disponible réel pour la boucherie, entre 11 et $12 \%$ et c'est, en réalité, le pourcentage utíle.

\section{Où vont ces animaux de boucherie?}

$1^{\circ}$ La consommation locale est 'd'environ 500.000 boeufs par an.

$2^{\circ}$ Les douze usines de conserves installées dans l'île ont abattu en 1948, 114.000 boufs, qui ont donné 4.800 tonnes de viande congelée el 7.000 tonnes de conserves diverses. 
$3^{\circ}$ Environ 10.000 bouls ont été exportés vivants sur l'île Maurice qui est tribulaire de Madagascar pour sa viande fraîche.

Si l'on ajoute aux considérations précédentes qu'il se fait entre les éleveurs un commerce très important de jeunes bœufs mis en réserve et engraissés pour une revente future, on peut voir quelle est l'importance de l'élevage du zébu à Madagascar.

\section{Amélioration}

Que faire pour l'amélioration du troupeau?

Le Service vétérinaire de Madagascar devenu le Service de l'Élevage et des Industries animales a eu comme but :

En premier lieu ne pas nuire. Fallait-il appliquer toutes les bonnes méthodes théoriques qui, avec une règle à calcul, font des animaux extraordinaires, souvent si extraordinaires qu'ils ne résistent plus dans le milieu auquel ils sont destinés.

Avant d'améliorer il a fallu penser à maintenir.

Maintenir : le nombre des zébus, leur rusticité, en les soustrayant aux épizooties mondiales.

Depuis l'arrivée des Français à Madagascar, et malgré l'importation d'animaux d'Europe ou d'Afrique du Sud, la fièvre aphteuse, la peste bovine. l'east coast fever, la péripneumonie et même la brucellose, ont pu être évitées. Les grands ennemis de notre élevage sont le charbon et la tuberculose. En ce qui concerne la piroplasmose er l'anaplasmose, une résistance naturelle est rencontrée chez le zébu, mais les jeunes veaux succombent souvent de heart water et de maladies vermineuses. Madagascar ignore les trypanosomiases Le charbon est efficacement tenu en échec par la vaccination systématique de 2 millions d'animaux par an dans les régions spécialement dangereuses. Ainsi se sont terminées les grandes épizooties d'avarıl 1900. L'éradication de la tuberculose se heurte à l'immensité du territoire et au mode d'entretien des animaux. Le Nord de l'île est resté pratiquement indemne grâce à des mesures sanitaires pertinentes (1 \% d'animaux tuberculeux à l'abattoir) et dans les autres régions, les lésions sont toujours légères sauf chez les bœufs de travail astreints à un effort constant.

La lutte contre les tiques est entreprise avec vigueur par la construction, très coûteuse cependant, de bains antiparasitaires. L'emploi de ces bains permettra de diminuer les cas de maladies dues aux tiques et évitera aux animaux de souffrir directement de la présence de ces parasites qui provoquent parfois de graves lésions cutanées pouvani aller jusqu'à la destruction des mamelles chez les vaches suiiées.

Quant à l'amélioration propremeñ dite, e.le n'est pas absolument nécessaire en ce qui concerne le zébu de boucherie qui est pour l'instant un animal présentant des qualités suffisantes. Des expériences de croisement avec différentes races ont été effectuées dans les stations gouvernementales, mais absolument rien dans le troupeau malgache. De ces expériences, il faut conclure que les' croisements de première génération qui sont d'un excellent format et qui conservent la rusticité du zébu seront à préconiser quand les conditions de nourriture auront éié améliorées, mais qu'il n'est guère possible de pousser plus ioin si le produit ne doit pas recevoir de soins spéciaux.

A l'arrivée des Français à Madagascar, il existait une variété de bœufs sans bosse appelée "rana » créée de toutes pièces par le Français Jean traborde qui, dès 1840, importa des taureaux, notamment des garonnais, des bordelais, et les croisa avec des zébus dans les régions des hauts-plateaux. Ces " Rana " se reproduisirent entre eux et les vaches sont d'assez bonnes laituères.

Soucieux dameliorer encore les « rana ", le Service de l'Elevage a importé jusqu'en 1939, 149 taureaux et 79 vaches de races diverses : mezenc, Schwytz, montbéliarde et surtout Normande.

Ces animaux sont entretenus dans les fermes du Service de 'Élevage. Les taureaux sont placés dans des haras et des stations de monte et mis gratuitement à la disposition des éleveurs qui amènent leurs vaches à la saillie dans ces Établissements.

Les produits issus des animaux de race pure deviennent à leur tour des géniteurs qui font des saillies gratuites.

Chaque année, pour encourager les éleveurs, le Service de l'Élevage achète les meilleurs taureaux do l'Élevage local et les utilise en leur, faisant saillir des vaches zébus.

Contrairement à ce que l'on aurait pu penser, les animaux de race normande qui vivent en France sur de très riches pâturages se sont comportés aussi bien, sinon mieux que les autres races importées. Ce sont maintenant les métis normands qui sont les plus nombreux et aussi les plus estimés pour leur rendement en lait.

Des expériences plus récentes sont poursuivies avec des "Friesland 》 importés de l'Afrique du Sud. Les résultats sont excellents, d'autant - et c'est une condition de succes - que ces animaux sont très appréciés des indigènes.

La multiplicité des races importées n'est pas due à un manque de suite dans l'amélioration du bétail local. Au contraire, elle s'explique par la nécessité d'étudier le comportement de diverses races amélioratrices, ainsi que le comportement des produits issus de leur croisement avec les zébus, dans le milieu malgache, de façon à pouvoir déterminer à 
l'avance la race à adopter lorsque l'amélioration générale des conditions du milieu permettra d'entreprendre l'amélioration, par croisement, du cheptel local.

C'est surtout sur les hauts-plateaux que s'est intensifié le croisement, mais le nombre des vaches métisses ne doit pas dépasser 25.000.

Le rendement laitier est très variable car l'éleveur indigène ne veut pas nourrir convenablement ses animaux. La lactation donne de 5 à 900 litres pour une durée de six à neuf mois. Lorsque ces vaches changent de propriétaire et obtiennent une nourriture convenable, les rendements sont augmentés de façon appréciable.

La laiterie municipale de Tamatave dirigée par un vétérinaire français, a pu doubler les rendements de vaches achetées sur les hauts-plateaux (alors que le climat de Tamatave est très chaud et très humide) en les nourrissant de façon rationnelle. A Tamatave, 30 vaches pour la plupart métisses normandes, en provenance des hauts-plateaux, ont donné en un an 57.000 litres de lait, soit une moyenne de 1.900 litres par tête et par an.

Les meilleurs rendements journaliers ont dépassé 20 litres.

La vache « Cavalière » a donné

3.182 litres en 465 jours.

La vache «Babiole » a donné

2.699 litres en 330 jours.

La vache « Caroline » a donné

2.810 litres en 300 jours.

La vache «Dandine » a donné

3.644 litres en 615 jours.

La meilleure vache « rana » a donné 2.200 litres en trois cents jours avec un maximum de 14 litres en un jour.

Le chiffre maxima pour les Hauts-Plateaux, en ce qui concerne les vaches métissées sont les suivants :

1 vache rana : "Jenny 》-2.800 litres en 300 jours. 1 vache métissée normande : "Dragée " 3.800 litres en 300 jours.

1 vache métissée normande "Canebière » 4.250 litres en 330 jours.

Tels sont les résultats auxquels est parvenu le Service de l'Élevage en partant du zébu pour arriver au métis en passant par la rana.

On peut transformer très rapidement le troupeau laitier par croisement continu et c'est le seul moyen d'arriver à un bon résultat. Il faut pour cela des reproducteurs, des soins et de la volonté.

Dans l'avenir, il est envisagé un centre de recherches zootechniques où seront étudiées les questions de génétique et de physiologie qui, jusqu'ici, n'ont pas fait à Madagascar l'objet d'études spéciales.
Dans un pays neuf, il a fallu faire vite et les méthodes expérimentales sur le terrain ont donné satisfaction.

En ce qui concerne l'amélioration du troupeau pour la viande, seule la sélection pourra donner des résultats féconds en milieu autochtone.

On peut résumer ainsi le plan d'amélioration pour l'avenir en ce qui concerne la production laitière :

$1^{\circ}$ Études au Centre de Recherches zootechniques;

20 Application dans les stations gouvernementales;

3o Importation des géniteurs améliorés et reproduction de ces géniteurs dans les fermes;

$4^{0}$ Mise à la disposition des éleveurs indigènes, gratuitement, de ces géniteurs:

$5^{\circ}$ Plus tard, distribution directement dans certains troupeaux indigènes.

Concurremment, amélioration des conditions de l'élevage par la constitution de réserves alimentaires et la construction de bains antiparasitaires.

Ce plan est déjà en application et sera de plus en plus ćtondu dans l'avenir.

\section{ÉLEVAGE DE LA ChÈVRE}

185.000 chèvres ont été recensées dans I'île. On les trouve le long de la côte ouest où elles servent comme animaux de boucherie aux populations musulmanes et surtout dans le Sud-Ouest et le Sud.

La chèvre malgache correspond nettement à deux types :

a) La chèvre de Nubie, à poil ras, au corps allongé, aux oreilles longues et pendantes, robe pie-rouge et pie-noir;

b) Un autre type plus difficile à déterminer comme origine : corps plus ramassé, plus osseux., couvert de longs poils raides et armé de cornes très développées.

Productivité. - Les chèvres malgaches ne sont utilisées ni pour le lait, ni pour la toison, celle-ci n'existant pas. Leur destination unique est la boucherie et leur chair n'est réellement appréciée que par la population musulmane ou indienne de la côte ouest. Dans le Sud, l'élevage est pratiqué par habitude ancestrale et la chair en est consommée également pour 'épargner normalement le sacrifice du « dieu-bœuf ».

Dans le Sud-Ouest, qui est lé.plus désertique, la chèvre est plus répandue parce que l'élevage du bceuf y est plus difficile qu'ailleurs, que le mouton n'y trouve que des herbages qui ne sont pas à sa convenance, parce que, surtout, la région est plus ou moins recouverte de buissons qui conviennent admirablement à son alimentation.

Sur le plateau central, la viande de chèvre à goût 
musqué n'est pas prisée. Mais l'emploi pour la laiterie aurait été susceptible de s'y développer, car le lait des chèvres est réputé chez l'autochtone comme une panacée contre les maladies de nutrition et la coqueluche.

Le Service vétérinaire avait, dans ce but, créé une ferme de chèvres laitières, de race alpine, en plein centre du massif montagneux de l'île. Cet élevage s'annonçait prospère. Il a dû être fermé par mesure d'économie et les animaux ont été dispersés dans l'élevage local, sans que nous ayons pu suivre leur comportement et leur évolution.

Introduction de races étrangères. - Les gens compétents réclamaient depuis longtemps l'introduction de I'angora dans le Sud. Celle-ci a été réalisée par le Gouvernement local, en 1913; avec quelques unités importées de la Colonie du Kénya.

La variété est africaine et se croise très facilement avec la race locale. Les animaux métis prennent les qualités de leurs ascendants paternels, mais celles-ci sont faibles en tant que production de mohair.

Aussi s'attacha-ton, en 1924, à amener d'Afrique du Sud un lot de 24 angoras sud afrique, à mohair fin, qui furent élevées à la ferme de Tuléar, dans le Sud-Ouest.

Cette variété s'acclimate facilement et montre une très grande facilité pour transmettre ses caractères à la variété malgache, si bien que le troupeau a fait boule de neige et qu'il comptait 130 têtes en 1929.

C'est dans le Sud-Ouest que se sont portés les efforts de notre Service pour faire du croisement continu et actuellement 5 fermes et stations distribuent des géniteurs aux éleveurs malgaches. Les résultats obtenus sont plus qu'encourageants.

En effet, alors que les premiers boucs angora ont été cédés aux éleveurs en 1930, on estime actuellement le nombre total de caprins de cette race à 30.000 têtes environ.

L'éleveur Mahafaly a rapidement compris toutes les ressources qu'il pouvait tirer de cet élevage. Lorsqu'il a été privé de tissus d'importation, il a filé et tissé le mohair de ses chèvres pour se faire des « lambas » et des couvertures. Et maintenant il fait même des tapis. Mais cette activité doit être dirigée et il reste beaucoup, sinon tout à faire, pour sortir, vendre et exporter des ouvrages finis capables de concurrencer ceux d'autres pays. Une Société Coopérative vient de se former. Elle aura non seulement des instructeurs, mais apposera son label sur les ouvrages correctement présentés.

La chèvre étant peu sensible aux gales et d'une rusticité proverbiale, nous sommes en droit d'espérer que cet élevage d'angoras ne fera que prospérer.

\section{ÉLEVAGE DU PORC}

Toutes les régions de la Grande lle sont propices à l'élevage du porc, et principalement les régions centrales (Tananarive, Antsirabé, Fianarantsoa).

Le porc malgache tire son origine de l'ancienne race ibérique introduite à Madagascar par les premiers navigateurs. Certains massifs montagneux du Centre, et en particulier celui de l'Ankaratra, offrent une variété autochtone différente, qui s'apparente à la race porcine chinoise.

Le porc malgache, entretenu par la population indigène, d'ure manière primitive et extrêmement précaire, a subi de ce fait une dégénérescence progressive.

C:est alors que, mettant a profit les conditions naturelles favorabies à la pullulation de l'espèce, les services techniques de Madagascar ont entrepris d'améliorer le type local par croisement avec des races importées :

Le craonnais donne des métis d'un gabarit considérable, mais manquant de rusticité et de résistance aux infestations parasitaires.

Le Yorkshire Middlewhite, excellent raceur, a conféré au porc local une aptitude excessive à l'engraissement, qui contrarie sa prolificité.

Actuellement le Service de l'Élevage s'oriente vers le croisement avec le Large White, qui paraît mieux adapté aux conditions économiques locales.

Le porc gras est très apprécié des peuplades indigènes de l'Imerina. Pendant longtemps, l'exploitation industrielle du porc malgache fut limitée à la préparation du saindoux. Depuis la guerre de 1914 , ce débouché s'est considérablement accru grâce à l'installation de plusieurs usines spécialement outillées pour le traitement et la conserve des viandes de bœuf et de porc ainsi que la préparation des produits de charcuterie.

Ces diverses préparations trouvent: un débouché régulier vers la consommation locale et vers l'exportation sur les pays voisins et aussi sur la métropole.

\section{ÉLEVAGE DU MOUTON}

180.000 moutons ont été recensés fin 1948 à Madagascar.

Par suite de la climatologie spéciale du pays, l'aire d'expansion favorable à l'espèce ovine est relativement réduite. Son élevage est, en effet, limité à quelques petites zones des plateaux centraux à climat tempéré où l'on dénombre 60.000 têtes et au Sud et Sud-Ouest, dont la nature subdésertique se prête le mieux à son développement, où vivent environ 120.000 moutons.

L'origine de la race est inconnue. On la croit cependant aussi ancienne que le bœuf autochtone 
et les premiers explorateurs signalent sa présence.

Elle appartient au type Ovis dolichura qui est plutôt intermédiaire entre la race d'Asie à queue courte et grosse Ovis statopygea et celle du Soudan à queue longue et tombante Ovis Africana.

Effectivement, le mouton malgache est caractérisé par une queue grosse, falciforme, longue, tombant sur les jarrets, dans laquelle l'accumulation adipeuse se fait aussi bien près de la pointe qu'à la base, près du sacrum

Par ailleurs, il rappelle beaucoup le mouton d'Égypte : tête longue à chanfrein busqué, oreilles longues et tombantes, cornes absentes ou petites à peine contournées et striées. La toison est uniquement constituée par du jarre, plus abondant à l'encolure, à l'épaule, sur le dos et la croupe, plus court et plus fin sur le ventre et les pattes. La robe est quelquefois entièrement rousse, plus rarement noire, mais la plus fréquente est le pie-noir et le pie-roux. La pigmentation noire ou rousse se localise souvent à la tête et à l'encolure à la façon du « Black head Persian $》$.

Aucun soin n'est donné à ces animaux qui vivent comme les bceufs, le plus souvent à l'aventure, et suivant ceux-ci à la recherche de leur nourriture.

Ils sont donc devenus très rustiques, mais le type ne s'est pas amélioré.

Le mouton malgache n'a ni une conformation favorable à la boucherie, ni une toison d'une valeur commerciale quelconque. La chair est filandreuse et de goût musqué, d'autant plus que l'éleveur ne le castre pas, à part quelques exceptions sur les plateaux.

Les brebis sont prolifiques et bonnes laitières; elles donnent fréquemment naissance à deux agneaux qu'elles élèvent fort bien.

Les poids moyens vifs varient entre 25 et 35 à 40 kilos sur les plateaux. Dans le Sud, il n'est pas rare de voir des mâles adultes atteindre 60 et même 80 kilos avec un rendement moyen de $50 \%$ environ à cause de la fincsso du squeletto.

\section{Productivité}

Le mouton malgache n'est utilisé que pour la boucherie. Dans le Sud, il est assez souvent abattu, comme la chèvre, pour les sacrifices à la place du boeuf trop gros, trop cher ou trop vénéré.

Sur les plateaux, le gros consommateur est la ville de Tananarive qui en absorbe près de 13.000 par an. Elle en consommerait davantage s'il était meilleur, c'est-à-dire amélioré. Mais l'éleveur ne s'est guère laissé tenter par cet élevage délicat ainsi que nous le montrerons plus loin.

\section{Introduction de races étrangères}

Par suite de la dégénérescence très avancée du mouton indigène notre Service a toujours estimé que l'amélioration ne pouvait se faire par sélection pure, mais uniquement par croisement.

Certains connaisseurs ont même prétendu que des régions étendues de la Grande Ile se prêtent suffisamment à l'élevage de l'espèce pour motiver l'importation directe d'une race améliorée et son exploitation unique au lieu et place de la race malgache.

Les aléas de cette dernière méthode étant suffisamment probants, le Gouvernement local a préféré l'amélioration par un croisement de géniteurs importés avec les brebis autochtones.

Mais il fallait trouver à la fois la race la plus apte à s'acclimater dans l'île et les lieux propices à son développement 'futur.

C'était s'imposer forcément des essais qui, jusqu'à ce jour, ne sont pas encore tout à fait concluants.

Le Service de l'Élevage et les particuliers ont introduit successivement : le mérinos de Rambouillet, dans le Nord, en 1896; le barbarin et le solognot, sur les plateaux centraux, en 1901; le mérinos de Provence en 1903, sur les Plateaux, dans le Sud-Ouest, en 1903 et en 1909 et dans le Sud, en 1924 ; le mérinos de l'Ariège sur le plateau central, en 1923 et 1924 ; le mérinos de Rambouillet dans le Sud, en 1912. Le southdown, dans le Sud-Ouest, en 1914; le dishley mérinos dans le Centre en 1929 ainsi que les béliers Ile-de-France en 1935 et, enfin, le mérinos sud-afrique, dans le Sud et le Sud-Ouest, en 1944 et 1945 .

De toutes ces races, seul le mérinos de Provence semhle se comporter à peu près convenablement sous toutes les latitudes.

On peut remarquer que la plupart des efforts ont porté sur l'acclimatement d'un mouton à laine fine (mérinos) que d'un mouton à rendement mixte (laine ft viande): Le fait est dû à l'intervention des autorités métropolitaines poussées par les usines à laine fine de Roubaix et Tourcoing qui recherchaient les moyens de libérer le commerce français des achats considérables effectués chaque année à l'étranger.

Mais les déboires essuyés par les Chambres de Commerce de ces deux villes dans leurs essais d'acclimatement direct de la race mérinos sudAfrique ont démontré qu'il ne fallait pas' procéder aussi rapidement ni.s'adresser à des races aussi sensibles.

Et le Service de l'Élevage a pensé qu'avant de servir la Métropole il fallait " lancer 》! l'élevage ovin dans l'île et fournir d'abord aux populations locales, non seulement de la bonne viande mais aussi une matière première, même grossière, apte à faire des vêtements chauds dont ont grand besoin les populations des hauts-plateaux. 
Le croisement continu a donc été pratiqué sur les plateaux pour alimenter surtout les abattoirs de Tananarive. Ce fut un échec dû surtout à la mentalité de l'éleveur autochtone qui a l'habitude du mouton à grosse queue et méprise les « moutons à queue de chien ").

Dans le Sud, l'élevage du mouton réussit parfaitement dans notre ferme d'Ambovombe. Mais beaucoup moins bien dans l'élevage local oủ les pasteurs qui appartiennent, eux, aux races les moins évoluées de Madagascar, ne comprennent rien aux soins à donner aux animaux améliorés.
Tandis que la chèvre résiste à la plupart des affections, le mouton est infiniment plus fragile. Il est très réceptif à toutes les gales, à la heart water inoculée par les nombreuses tiques,' à toutes les infestations du tube digestif, etc.

Dans les conditions naturelles du pays, l'élevage du mouton amélioré a jusqu'ici été un échec. Il ne pourra donner des résultats probants qu'avec la multiplication des bains antiparasitaires, le traitement et la prophylaxie du parasitisme intestinal et une meilleure discipline de la part des éleveurs, et ceci n'est pas absolument certain. 